\title{
A METHOD OF LINES FOR A NONLINEAR ABSTRACT FUNCTIONAL EVOLUTION EQUATION
}

\author{
BY
}

\author{
A. G. KARTSATOS AND M. E. PARROTT
}

ABSTRACT. Let $X$ be a real Banach space with $X^{*}$ uniformly convex. A method of lines is introduced and developed for the abstract functional problem

$$
u^{\prime}(t)+A(t) u(t)=G\left(t, u_{t}\right), \quad u_{0}=\phi, \quad t \in[0, T]
$$

The operators $A(t): D \subset X \rightarrow X$ are m-accretive and $G(t, \phi)$ is a global Lipschitzian-like function in its two variables. Further conditions are given for the convergence of the method to a strong solution of (E). Recent results for perturbed abstract ordinary equations are substantially improved. The method applies also to large classes of functional parabolic problems as well as problems of integral perturbations. The method is straightforward because it avoids the introduction of the operators $\hat{A}(t)$ and the corresponding use of nonlinear evolution operator theory.

1. Introduction-Preliminaries. Let $X$ be a real Banach space with norm $\|\cdot\|$. Let PC be the space of piecewise continuous functions $\psi:[-r, 0] \rightarrow X$, for a fixed $r>0$. For $\psi \in \mathrm{PC}$ we let

$$
\|\psi\|_{\mathrm{PC}}=\sup _{s \in[-r .0]}\|\psi(s)\| .
$$

In this paper we consider the abstract nonlinear functional problem

$$
u^{\prime}(t)+A(t) u(t)=G\left(t, u_{t}\right), \quad u_{0}=\phi, \quad t \in[0, T],
$$

where $u:[-r, T] \rightarrow X, A(t): D \subset X \rightarrow X, t \in[0, T], D$ independent of $t, G:[0, T] \times$ PC $\rightarrow X$, and $\phi:[-r, 0] \rightarrow X$ is Lipschitzian with $\phi(0) \in D$. The symbol $u_{t}$ denotes the function $u_{t}(\theta)=u(t+\theta), \theta \in[-r, 0]$. We also need the following conditions:

(A.1) $X^{*}$, the dual of $X$, is uniformly convex.

(A.2) There exists a nondecreasing function $L_{1}:[0, \infty) \rightarrow[0, \infty)$ such that

$$
\|A(t) x-A(s) x\| \leqslant|t-s| L_{1}(\|x\|)(1+\|A(s) x\|)
$$

for every $s, t \in[0, T]$ and every $x \in D$.

(A.3) For each $t \in[0, T], A(t)$ is $m$-accretive (see definition below).

(A.4) There exists a constant $\beta>0$ such that, for $\phi, \psi \in \mathrm{PC}, t \in[0, T]$,

$$
\|G(t, \phi)-G(t, \psi)\| \leqslant \beta\|\phi-\psi\|_{\mathrm{PC}} .
$$

Received by the editors June 10, 1982.

1980 Mathematics Subject Classification. Primary 34H05, 43A75.

Key words and phrases. Functional evolution equation, uniformly convex dual, method of lines, $m$-accretive operator. 
(A.5) There exists $L_{2}:[0, \infty) \rightarrow[0, \infty)$ nondecreasing and such that

$$
\|G(t, \phi)-G(s, \phi)\| \leqslant L_{2}\left(\|\phi\|_{\mathrm{PC}}\right)|t-s|
$$

for every $s, t \in[0, T], \phi \in$ PC.

Let $\langle x, f\rangle$ denote the value of the functional $f \in X^{*}$ at $x \in X$. Define the "duality map" on $X$ as follows:

$$
J x=\left\{x^{*} \in X^{*} ;\left\langle x, x^{*}\right\rangle=\|x\|^{2}=\left\|x^{*}\right\|^{2}\right\} .
$$

The set $J x$ is nonempty for each $x \in X$ by the Hahn-Banach theorem. If $X^{*}$ is uniformly convex, then $J$ is single valued and uniformly continuous on bounded subsets of $X$. An operator $B: D(B) \subset X \rightarrow X$ is called "accretive" if for every $x$, $y \in D(B)$ there exists $f \in J(x-y)$ such that $\langle B x-B y, f\rangle \geqslant 0$.

An accretive operator $B$ is called " $m$-accretive" if $R(I+\lambda B)=X$ for some (equivalently, for all) real $\lambda>0$. If $B$ is $m$-accretive, it can be shown that, for any $\lambda>0$, the operator $(B+\lambda I)^{-1}$ exists and, for every $u, v \in X$, satisfies

$$
\left\|(B+\lambda I)^{-1} u-(B+\lambda I)^{-1} v\right\| \leqslant(1 / \lambda)\|u-v\| .
$$

For these and other properties of accretive operators we refer the reader to Kato [12]. By a "strong solution" of (FDE) we mean a continuous function $u$ : $[-r, T] \rightarrow X$ which is absolutely continuous on $[0, T]$ and satisfies (FDE) (strongly) almost everywhere there.

Crandall and Pazy [3] established the existence of solutions of an abstract evolution equation in a general Banach space. The equation they considered was of the form

$$
u^{\prime}(t)+A(t) u(t) \ni 0, \quad u(s)=x, \quad s \leqslant t \leqslant T,
$$

where $A(t)$ is a nonlinear (possibly multivalued) operator satisfying an $m$-accretiveness-type condition and a time dependence condition implied by (A.2). Moreover, they assumed that $\overline{D(A(t))}$ is independent of $t$. The existence results in [3] are based on the existence and the properties of abstract evolution operators generated by such operators $A(t)$.

In [18] Webb showed that the autonomous version of (FDE) can be studied in the framework of nonlinear semigroup theory $(A(t)=A$ in (AE)) by defining an operator $\hat{A}: D(\hat{A}) \subset C \rightarrow C(=$ the space of continuous functions $f:[-r, 0] \rightarrow X)$ as follows:

$$
\hat{A \phi}=-\phi^{\prime}
$$

with

$$
D(\hat{A})=\left\{\phi \in C ; \phi^{\prime} \in C, \phi(0) \in D(A), \phi^{\prime-}(0)+A \phi(0)=G(\phi)\right\} .
$$

In [5 and 6] Dyson and Villella Bressan extended Webb's approach to the nonautonomous equation (FDE) under conditions similar to (A.2)-(A.5) and for $X^{*}$ uniformly convex.

One advantage in considering (FDE) as the abstract "homogeneous" equation

$$
u^{\prime}(t)+\hat{A}(t) u(t)=0, \quad u(0)=\phi,
$$


is that the approximation theory of Crandall and Pazy for (AE) (see also $[7,8,13]$ ) can now be applied to (FDE). In [15] Parrott indicates exactly how this can be accomplished.

In [10] the authors established the fact that the unique solution of (FDE) (in spaces with $X^{*}$ uniformly convex) can actually be obtained in a straightforward manner without the introduction of the operator $\hat{A}(t)$ above and without the use of nonlinear evolution operator theory. In fact, it was shown in [10] that this solution $u(t)$ is the uniform limit of solutions $u_{n}(t)$ of approximate equations involving the Yosida approximants of $A(t)$.

We should also mention here that the problem

$$
u^{\prime}(t)=f\left(t, u_{t}\right), \quad u_{t_{0}}=\phi, \quad t \geqslant t_{0},
$$

(or its autonomous counterpart) has been solved by use of evolution operator theory in [19] among others. The corresponding integral equation has been considered in [9]. In the case $X=R^{n}$, the numerical approximation of (F) has been accomplished by a variety of methods (see, for example, [4] and the references therein). Linear semigroup theory for the approximation of linear hereditary systems has been utilized by Banks and Burns [1], Banks and Kappel [2], Thompson [17] and some of the references therein.

It is our purpose in this paper to introduce and develop a "method of lines" approximation scheme for solutions of (FDE). The method of lines approximating parabolic problems goes back to Rothe [16]. This method was used by Nečas [14] to study the problem

$$
u^{\prime}(t)+A u(t)=Q(t), \quad u(0)=u_{0}, \quad t \in[0, T],
$$

with $X$ a Hilbert space. The results of Nečas were extended by Kartsatos and Zigler in [11], where the method of lines was used in order to establish the existence and uniqueness of the solution of the perturbed evolution equation

$$
u^{\prime}(t)+A(t) u(t)=G(t, u(t)), \quad u(0)=u_{0}, \quad t \in[0, T],
$$

in a Banach space $X$ with $X^{*}$ uniformly convex. Since (FDE) includes (PE) as a special case, our results constitute a substantial improvement of certain results of [11].

The approximation scheme developed here has the advantage of being straightforward in the sense that we need not resort to the operators $\hat{A}(t)$ nor further restrict the space of initial functions $\phi$.

We should also mention that it is impossible to apply evolution operator theory to our problem without defining the rather impractical operators $\hat{A}(t)$ and solving the corresponding initial value problem.

2. The approximation method. As noted in $\$ 1$, the existence of a unique strong solution $u(t)$ of (FDE) under assumptions (A.1)-(A.5) has previously been established. We will construct $u(t)$ as the uniform limit of "lines" which are the solutions of approximate discrete equations for (FDE). In what follows, we assume that conditions (A.1)-(A.5) hold.

We consider a partition $\left\{t_{n j}\right\}$ of the interval $[0, T]$, where $t_{n j}=j h=j T / n$, $j=0,1, \ldots, n\left(t_{n 0}=0, t_{n n}=T\right)$. We assume that $n$ is sufficiently large so that 
$b h<1$, where $b$ is the constant of condition (A.4). We set

$$
\bar{z}_{n 0}(t)= \begin{cases}\phi(t), & t \in[-r, 0] \\ \phi(0), & t \in[0, T] .\end{cases}
$$

We also define the space

$$
\begin{aligned}
B_{1}=\{u:[-r, T] \rightarrow X ; u(t)=\phi(t) & \text { for } t \in[-r, 0] \\
& \text { and } u(t) \text { is constant on }(0, T]\} .
\end{aligned}
$$

It is easy to check that $B_{1}$ is a complete metric space with respect to the distance function induced by the norm

$$
\|u\|_{T}=\sup _{t \in[-r . T]}\|u(t)\|_{1}
$$

We define a mapping $U_{1}$ on $B_{1}$ such that

$$
\left(U_{1} u\right)(t)=\left\{\begin{array}{l}
\phi(t), \quad t \in[-r, 0], \\
\left(A\left(t_{n 0}\right)+(1 / h) I\right)^{-1}\left(z_{n 0} / h+G\left(t_{n 0}, u_{t_{n 0}}\right)\right), \quad t \in(0, T],
\end{array}\right.
$$

where $z_{n 0}=\phi(0)$. The mapping $U_{1}: B_{1} \rightarrow B_{1}$ is a strict contraction on $B_{1}$. In fact, for $u, v \in B_{1}$,

$$
\begin{aligned}
\left\|U_{1} u-U_{1} v\right\|_{T} & \leqslant h\left\|G\left(t_{n 0}, u_{t_{n 0}}\right)-G\left(t_{n 0}, v_{t_{n 0}}\right)\right\| \leqslant h \beta\left\|u_{t_{n 0}}-v_{t_{n 0}}\right\|_{\mathrm{PC}} \\
& \leqslant h \beta \sup _{\theta \in[-r .0]}\left\|u_{t_{n 0}}(\theta)-v_{t_{n 0}}(\theta)\right\|=h \beta \sup _{t \in\left[t_{n 0}-r, t_{n 0}\right]}\|u(t)-v(t)\| \\
& \leqslant h \beta \sup _{t \in[-r, T]}\|u(t)-v(t)\|=h \beta\|u-v\|_{T} .
\end{aligned}
$$

We denote by $\bar{z}_{n 1}$ the unique fixed point of $U_{1}$ on $B_{1}$. We also let

$$
z_{n 1}=\left(A\left(t_{n 0}\right)+(1 / h) I\right)^{-1}\left(z_{n 0} / h+G\left(t_{n 0}, \bar{z}_{n 1 n 0}\right)\right) \text {. }
$$

Proceeding in a similar manner we introduce the spaces $B_{j}, j=1,2, \ldots, n$, by

$$
\begin{aligned}
B_{j}=\{u & :[-r, T] \rightarrow X ; u(t)=\phi(t) \text { for } t \in[-r, 0], \\
& \left.u(t) \text { is constant on each interval }\left(0, t_{n 1}\right],\left(t_{n 1}, t_{n 2}\right], \ldots,\left(t_{n, j-1}, T\right]\right\} .
\end{aligned}
$$

Each space $B_{j}$ is a complete metric space with the same distance function as $B_{1}$. Moreover, the strict contractions $U_{j}: B_{j} \rightarrow B_{j}$ are defined by

$$
\left(U_{j} u\right)(t)= \begin{cases}\phi(t), & t \in[-r, 0], \\ z_{n 1}, & t \in\left(0, t_{n 1}\right], \\ z_{n 2}, & t \in\left(t_{n 1}, t_{n 2}\right], \\ \vdots & \vdots \\ z_{n, j-1}, & t \in\left(t_{n, j-2}, t_{n, j-1}\right], \\ \left(A\left(t_{n, j-1}\right)+(1 / h) I\right)^{-1}\left(z_{n, j-1} / h+G\left(t_{n, j-1}, u_{t_{n, j-1}}\right)\right), & t \in\left(t_{n, j-1}, T\right] .\end{cases}
$$


The unique fixed point of $U_{j}$ on $B_{j}$ is denoted by $\bar{z}_{n j}$ and we let

$$
\begin{aligned}
& z_{n j}=\left(A\left(t_{n, j-1}\right)+(1 / h) I\right)^{-1}\left(z_{n, j-1} / h+G\left(t_{n, j-1}, \bar{z}_{n j_{t n,-1}}\right)\right) \text {, } \\
& j=1, \ldots, n \text {. }
\end{aligned}
$$

From (2.4) we see that for each $j=1,2, \ldots, n$ the point $z_{n j}$ satisfies

$$
A\left(t_{n, j-1}\right) z_{n j}+\left(z_{n j}-z_{n, j-1}\right) / h=G\left(t_{n, j-1}, \bar{z}_{n j_{i_{n, j-1}}}\right) \text {. }
$$

LEMMA 2.1. The sequence $\left\{z_{n j}\right\}$ is uniformly bounded.

Proof. We first establish the fact that, without loss of generality, we may assume that $0 \in D$ and $A(t) 0=0$ for $t \in[0, T]$. In fact, if $0 \notin D$, we fix $x_{0} \in D$ and define $A_{1}(t)$ by $A_{1}(t) x=A(t)\left(x+x_{0}\right)-A(t) x_{0}$ for every $x \in D-x_{0}=D_{1}$. Then $0 \in D_{1}$ and $A_{1}(t) 0=0$. Clearly, $A_{1}(t)$ is $m$-accretive since $A(t)$ is $m$-accretive. Also, if we define the constant function $\bar{x}_{0} \in \mathrm{PC}$ by $\bar{x}_{0}(\theta)=x_{0}$ for $\theta \in[-r, 0]$, then we can write (FDE) as

$$
\begin{aligned}
u^{\prime}(t)+A_{1}(t) u(t) & =u^{\prime}(t)+A(t)\left(u(t)+x_{0}\right)-A(t) x_{0} \\
& =G\left(t, u_{t}+\bar{x}_{0}\right)-A(t) x_{0} \stackrel{\text { def }}{=} G_{1}\left(t, u_{t}\right)
\end{aligned}
$$

We show that $A_{1}(t)$ satisfies a time-dependence condition of the form (A.2). Let $t, s \in[0, T]$ and $x \in D_{1}$ be given. Then we have

$$
\begin{aligned}
A_{1}(t) x-A_{1}(s) x= & A(t)\left(x+x_{0}\right)-A(t) x_{0} \\
& -\left[A(s)\left(x+x_{0}\right)-A(s) x_{0}\right],
\end{aligned}
$$

which implies

$$
\begin{aligned}
\left\|A_{1}(t) x-A_{1}(s) x\right\| \leqslant & \left\|A(t)\left(x+x_{0}\right)-A(s)\left(x+x_{0}\right)\right\| \\
& +\left\|A(t) x_{0}-A(s) x_{0}\right\| \\
\leqslant & |t-s| L_{1}\left(\left\|x+x_{0}\right\|\right)\left(1+\left\|A(s)\left(x+x_{0}\right)\right\|\right) \\
& \quad+|t-s| L_{1}\left(\left\|x_{0}\right\|\right)\left(1+\left\|A(s) x_{0}\right\|\right) \\
\leqslant & |t-s| L_{3}(\|x\|)\left(2+\left\|A(s)\left(x+x_{0}\right)\right\|+\left\|A(s) x_{0}\right\|\right),
\end{aligned}
$$

where $L_{3}(\|x\|)=\max \left\{L_{1}\left(\|x\|+\left\|x_{0}\right\|\right), L_{1}\left(\left\|x_{0}\right\|\right)\right\}$. Since

$$
\begin{aligned}
\left\|A(s)\left(x+x_{0}\right)\right\| & =\left\|A(s)\left(x+x_{0}\right)-A(s) x_{0}+A(s) x_{0}\right\| \\
& \leqslant\left\|A_{1}(s) x\right\|+\left\|A(s) x_{0}\right\|,
\end{aligned}
$$

we obtain

$$
\left\|A_{1}(t) x-A_{1}(s) x\right\| \leqslant|t-s| L_{3}(\|x\|)\left(2+\left\|A_{1}(s) x\right\|+2\left\|A(s) x_{0}\right\|\right) .
$$

Since (A.2) implies that $\left\|A(s) x_{0}\right\|$ is bounded on $[0, T]$, the above inequality implies that

$$
\left\|A_{1}(t) x-A_{1}(s) x\right\| \leqslant K_{0}|t-s| L_{3}(\|x\|)\left(1+\left\|A_{1}(s) x\right\|\right)
$$


for some $K_{0}>0$, which is condition (A.2) with $K_{0} L_{3}$ replacing $L_{1}$ and $A_{1}$ replacing $A$. The function $G_{1}(t, \phi)=G\left(t, \phi+\bar{x}_{0}\right)-A(t) x_{0}$ is Lipschitz continuous in $\phi \in \mathrm{PC}$ since $G$ has this property. Also, for $\phi \in \mathrm{PC}, s, t \in[0, T]$, we have

$$
\begin{aligned}
\left\|G_{1}(t, \phi)-G_{1}(s, \phi)\right\| & =\left\|G\left(t, \phi+\bar{x}_{0}\right)-A(t) x_{0}-G\left(s, \phi+\bar{x}_{0}\right)+A(s) x_{0}\right\| \\
& \leqslant|t-s| L_{2}\left(\left\|\phi+\bar{x}_{0}\right\|_{\mathrm{PC}}\right)+|t-s| L_{1}\left(\left\|x_{0}\right\|\right)\left(1+\left\|A(s) x_{0}\right\|\right) \\
& \leqslant L_{4}\left(\|\phi\|_{\mathrm{PC}}\right)|t-s| .
\end{aligned}
$$

where $L_{4}(u)=\max \left\{L_{2}\left(u+\left\|x_{0}\right\|\right), K_{1} L_{1}\left(\left\|x_{0}\right\|\right)\right\}$, for a suitable constant $K_{1}>0$. Now, using the Lipschitz continuity of $\left(A\left(t_{n, j-1}\right)+(1 / h) I\right)^{-1}$ and assuming that $A(t) 0=0$, we obtain from (2.3) and (2.4), for $t \in\left(t_{n, j-1}, T\right]$,

$$
\left\|\bar{z}_{n j}(t)\right\|=\left\|z_{n j}\right\| \leqslant\left\|z_{n, j-1}\right\|+h\left\|G\left(t_{n, j-1}, \bar{z}_{n j_{i_{n,-1}}}\right)\right\| .
$$

If we denote by $\overline{0}$ the zero function in PC and let $K_{2}$ be a bound for $\|G(t, \overline{0})\|$ on $[0, T]$, we obtain

$$
\begin{aligned}
\left\|\bar{z}_{n j}(t)\right\| & \leqslant\left\|z_{n, j-1}\right\|+h\left\|G\left(t_{n, j-1}, \bar{z}_{n j_{t_{n,-1}}}\right)-G\left(t_{n, j-1}, \overline{0}\right)\right\|+h\left\|G\left(t_{n, j-1}, \overline{0}\right)\right\| \\
& \leqslant\left\|z_{n, j-1}\right\|+h \beta\left\|\bar{z}_{n j_{t_{n, j-1}}}\right\| \|_{\mathrm{PC}}+h K_{2} .
\end{aligned}
$$

Now,

$$
\begin{aligned}
\left\|\bar{z}_{n j_{t_{n, j}, 1}}\right\| \mathrm{PC} & =\sup _{\theta \in[-r .0]}\left\|\bar{z}_{n j}\left(t_{n, j-1}+\theta\right)\right\| \\
& =\sup _{t \in\left[t_{n, j-1}-r . t_{n, 1}, 1\right]}\left\|\bar{z}_{n j}(t)\right\| \leqslant \sup _{t \in[-r . T]}\left\|\bar{z}_{n j}(t)\right\| .
\end{aligned}
$$

Hence,

$$
\begin{aligned}
\left\|\bar{z}_{n j}(t)\right\| & \leqslant \sup _{t \in[-r, T]}\left\|\bar{z}_{n, j-1}(t)\right\|+h \beta \sup _{t \in[-r . T]}\left\|\bar{z}_{n j}(t)\right\|+h K_{2} \\
& =\left\|\bar{z}_{n, j-1}\right\|_{T}+h \beta\left\|\bar{z}_{n j}\right\|_{T}+h K_{2} .
\end{aligned}
$$

If $t \in\left[-r, t_{n, j-1}\right]$, then $\left\|\bar{z}_{n j}(t)\right\|=\left\|\bar{z}_{n, j-1}(t)\right\| \leqslant\left\|\bar{z}_{n, j-1}\right\|_{T}$. Thus, for all $t \in[-r, T]$,

$$
\left\|\bar{z}_{n j}\right\|_{T} \leqslant\left\|\bar{z}_{n, j-1}\right\|_{T}+\beta h\left\|\bar{z}_{n j}\right\|_{T}+h K_{2},
$$

which yields

$$
(1-\beta h)\left\|\bar{z}_{n j}\right\|_{T} \leqslant\left\|\bar{z}_{n, j-1}\right\|_{T}+h K_{2}, \quad j=1,2, \ldots n .
$$

Applying the above inequality once more, we get

$$
\begin{aligned}
(1-\beta h)\left\|z_{n j}\right\|_{T} \leqslant & \frac{\left\|\bar{z}_{n, j-2}\right\|_{T}}{1-\beta h}+\frac{h K_{2}}{1-\beta h}+h K_{2} \\
& \vdots \\
& \leqslant \frac{\left\|\bar{z}_{n 0}\right\|_{T}}{(1-\beta h)^{n-1}}+\sum_{k=0}^{n-1}\left[\frac{h K_{2}}{(1-\beta h)^{k}}\right] .
\end{aligned}
$$


Thus

$$
\begin{aligned}
\left\|\bar{z}_{n j}\right\|_{T} & \leqslant \frac{\left\|\bar{z}_{n 0}\right\|_{T}}{(1-\beta h)^{n}}+\sum_{k=1}^{n}\left[\frac{h K_{2}}{(1-\beta h)^{k}}\right] \\
& \leqslant \frac{\|\phi\|_{\mathrm{PC}}}{(1-\beta h)^{n}}+\sum_{k=1}^{n}\left[\frac{h K_{2}}{(1-\beta h)^{n}}\right] \\
& =\frac{\|\phi\|_{\mathrm{PC}}}{(1-\beta T / n)^{n}}+\frac{T K_{2}}{(1-\beta T / n)^{n}} .
\end{aligned}
$$

Since $\lim _{n \rightarrow x} 1 /(1-\beta T / n)^{n}=e^{\beta T}$, we conclude there exists $n_{0}$ and $M>0$ such that for $n \geqslant n_{0}$ we have

$$
\left\|z_{n j}\right\| \leqslant\left\|\bar{z}_{n j}\right\|_{T} \leqslant M, \quad j=0,1, \ldots, n .
$$

LEMMA 2.2. The sequence $\left\{\left(z_{n j}-z_{n, j-1}\right) / h\right\}$ is uniformly bounded.

Proof. Assume that $n_{0}$ and $M$ are as in the proof of Lemma 2.1. Assume further that $\left(\beta+L_{1}(M)\right) h<1$ for $n \geqslant n_{0}$. From equation (2.5) we have, for $j=1$,

$$
A\left(t_{n 0}\right) z_{n 1}+\left(z_{n 1}-z_{n 0}\right) / h=G\left(t_{n 0}, \bar{z}_{n 1_{t_{n}}}\right) .
$$

It follows that

$$
\begin{aligned}
\left\langle A(0) z_{n 1}-A(0) z_{n 0},\right. & \left.J\left(z_{n 1}-z_{n 0}\right)\right\rangle+\left\|z_{n 1}-z_{n 0}\right\|^{2} / h \\
= & \left\langle G\left(0, \bar{z}_{n 1}\right), J\left(z_{n 1}-z_{n 0}\right)\right\rangle-\left\langle A(0) z_{n 0}, J\left(z_{n 1}-z_{n 0}\right)\right\rangle,
\end{aligned}
$$

which, using the accretiveness of $A(0)$, gives

$$
\begin{aligned}
\left\|z_{n 1}-z_{n 0}\right\|^{2} / h \leqslant & \left\langle G\left(0, \bar{z}_{n 1_{0}}\right)-G(0, \overline{0}), J\left(z_{n 1}-z_{n 0}\right)\right\rangle \\
& +\left\langle G(0, \overline{0}), J\left(z_{n 1}-z_{n 0}\right)\right\rangle-\left\langle A(0) z_{n 0}, J\left(z_{n 1}-z_{n 0}\right)\right\rangle \\
\leqslant & \beta\left\|\bar{z}_{n 1_{0}}\right\|_{\mathrm{PC}}\left\|z_{n 1}-z_{n 0}\right\|+K_{2}\left\|z_{n 1}-z_{n 0}\right\|+\left\|A(0) z_{n 0}\right\|\left\|z_{n 1}-z_{n 0}\right\| \\
\leqslant & {\left[\beta\|\phi\|_{\mathrm{PC}}+K_{2}+\|A(0) \phi(0)\|\right]\left\|z_{n 1}-z_{n 0}\right\|, }
\end{aligned}
$$

where $K_{2}$ is the upper bound of $\|G(t, \overline{0})\|$ from the proof of Lemma 2.1. Consequently, we obtain

$$
\left\|z_{n 1}-z_{n 0}\right\| / h \leqslant K_{3},
$$

where $K_{3}=\beta\|\phi\|_{\mathrm{PC}}+K_{2}+\|A(0) \phi(0)\|$. From (2.5) with $j=2,3, \ldots, n$ we find

$$
\begin{aligned}
& \left\langle A\left(t_{n, j-1}\right) z_{n j}-A\left(t_{n, j-2}\right) z_{n, j-1}, J\left(z_{n j}-z_{n . j-1}\right)\right\rangle+\left\|z_{n j}-z_{n, j-1}\right\|^{2} / h \\
& \quad=\left\langle G\left(t_{n, j-1}, \bar{z}_{n j_{t_{n, j-1}}}\right), J\left(z_{n j}-z_{n, j-1}\right)\right\rangle-\left\langle A\left(t_{n, j-2}\right) z_{n, j-1}, J\left(z_{n j}-z_{n, j-1}\right)\right\rangle .
\end{aligned}
$$


Hence, by the accretiveness of $A\left(t_{n, j-2}\right)$ and condition (A.2),

$$
\begin{aligned}
\left\|z_{n j}-z_{n, j-1}\right\|^{2} / h \leqslant & \left\langle G\left(t_{n, j-1}, \bar{z}_{n j_{t_{n, j}}}\right), J\left(z_{n j}-z_{n, j-1}\right)\right\rangle \\
& -\left\langle A\left(t_{n, j-2}\right) z_{n, j-1}, J\left(z_{n j}-z_{n, j-1}\right)\right\rangle \\
& -\left\langle A\left(t_{n, j-1}\right) z_{n j}-A\left(t_{n, j-2}\right) z_{n j}, J\left(z_{n j}-z_{n, j-1}\right)\right\rangle \\
& -\left\langle A\left(t_{n, j-2}\right) z_{n j}-A\left(t_{n, j-2}\right) z_{n, j-1}, J\left(z_{n j}-z_{n, j-1}\right)\right\rangle \\
\leqslant & \left\langle G\left(t_{n, j-1}, \bar{z}_{n j_{t_{n, j}}}\right), J\left(z_{n j}-z_{n, j-1}\right)\right\rangle \\
& -\left\langle A\left(t_{n, j-2}\right) z_{n, j-1}, J\left(z_{n j}-z_{n, j-1}\right)\right\rangle \\
& +\left|t_{n, j-1}-t_{n, j-2}\right| L_{1}\left(\left\|z_{n j}\right\|\right) \\
& \cdot\left(1+\left\|A\left(t_{n, j-1}\right) z_{n j}\right\|\right)\left\|z_{n j}-z_{n, j-1}\right\| .
\end{aligned}
$$

Since

$$
G\left(t_{n, j-1}, \bar{z}_{n j_{t_{n, j-1}}}\right)=G\left(t_{n, j-1}, \bar{z}_{n j_{t_{n, j-1}}}\right)-G\left(t_{n, j-2}, \bar{z}_{n j_{t_{n, j-2}}}\right)+G\left(t_{n, j-2}, \bar{z}_{n j_{t_{n, j-2}}}\right),
$$

and, for all $\theta \in[-r, 0]$,

$$
\bar{z}_{n j_{t_{n, j-2}}}(\theta)=\bar{z}_{n j}\left(t_{n, j-2}+\theta\right)=\bar{z}_{n, j-1}\left(t_{n, j-2}+\theta\right)=\bar{z}_{n, j-1_{t_{n, j-2}}}(\theta),
$$

we have the appraisals

$$
\begin{aligned}
& \left\|z_{n j}-z_{n, j-1}\right\|^{2} / h \leqslant\left\|G\left(t_{n, j-1}, \bar{z}_{n j_{t_{n, j-1}}}\right)-G\left(t_{n, j-2}, \bar{z}_{n j_{t_{n, j-2}}}\right)\right\|\left\|z_{n j}-z_{n, j-1}\right\| \\
& +\left\langle G\left(t_{n, j-2}, \bar{z}_{n, j-1_{i_{,-2}}}\right)-A\left(t_{n, j-2}\right) z_{n, j-1}, J\left(z_{n j}-z_{n, j-1}\right)\right\rangle \\
& +\left|t_{n, j-1}-t_{n, j-2}\right| L_{1}\left(\left\|z_{n j}\right\|\right)\left(1+\left\|A\left(t_{n, j-1}\right) z_{n j}\right\|\right)\left\|z_{n j}-z_{n, j-1}\right\| \\
& \leqslant\left\|G\left(t_{n, j-1}, \bar{z}_{n j_{t_{n, j-1}}}\right)-G\left(t_{n, j-1}, \bar{z}_{n j_{t_{n, j-2}}}\right)\right\|\left\|z_{n j}-z_{n, j-1}\right\| \\
& +\left\|G\left(t_{n, j-1}, \bar{z}_{n j_{i_{n, j-2}}}\right)-G\left(t_{n, j-2}, \bar{z}_{n j_{i_{n, j-2}}}\right)\right\|\left\|z_{n j}-z_{n, j-1}\right\| \\
& +\left\|z_{n, j-1}-z_{n, j-2}\right\|\left\|z_{n j}-z_{n, j-1}\right\| / h \\
& +h L_{1}(M)\left(1+\left\|z_{n j}-z_{n, j-1}\right\| / h+\left\|G\left(t_{n, j-1}, \bar{z}_{n j_{t_{n, j}-1}}\right)\right\|\left\|z_{n j}-z_{n, j-1}\right\|\right),
\end{aligned}
$$

from which it follows that

$$
\begin{aligned}
\left\|z_{n j}-z_{n, j-1}\right\| / h \leqslant \beta\left\|\bar{z}_{n j_{i_{n, j-1}}}-\bar{z}_{n j_{t_{n, j-2}}}\right\| \mathrm{PC} & +L_{2}(M)\left|t_{n, j-1}-t_{n, j-2}\right| \\
& +L_{1}(M)\left\|z_{n j}-z_{n, j-1}\right\|+h L_{1}(M) K_{4}+\left\|z_{n, j-1}-z_{n, j-2}\right\| / h,
\end{aligned}
$$

where

$$
1+\beta\left\|\bar{z}_{n_{t_{n, j}-1}}\right\| \mathrm{PC}+K_{2} \leqslant K_{4} .
$$


In order to find a suitable upper bound for

$$
\left\|\bar{z}_{n j_{t_{n, j-1}}}-\bar{z}_{n j_{i_{n, j-2}}}\right\| \mathrm{PC}=\sup _{\theta \in[-r, 0]}\left\|\bar{z}_{n j}\left(t_{n, j-1}+\theta\right)-\bar{z}_{n j}\left(t_{n, j-2}+\theta\right)\right\|
$$

we distinguish three cases.

Case $1 . t_{n, j-2} \geqslant r$.

We have $t_{n, j-1} \geqslant r$ and, for all $\theta \in[-r, 0]$,

$$
\begin{aligned}
\left\|\bar{z}_{n j}\left(t_{n, j-1}+\theta\right)-\bar{z}_{n j}\left(t_{n, j-2}+\theta\right)\right\| & =\left\|\bar{z}_{n j}\left(t_{n, j-1}+\theta\right)-\bar{z}_{n, j-1}\left(t_{n, j-2}+\theta\right)\right\| \\
& \leqslant \max _{1 \leqslant k \leqslant j}\left\|z_{n k}-z_{n, k-1}\right\| .
\end{aligned}
$$

Case 2. $t_{n, j-1}<r$.

We also have $t_{n, j-2}<r$. (a) For $\theta \in\left[-r,-t_{n, j-1}\right]$, we have $t_{n, j-1}+\theta \in\left[t_{n, j-1}-\right.$ $r, 0]$ and $t_{n, j-2}+\theta \in\left[t_{n, j-2}-r, 0\right]$. Denoting by $L_{0}$ the Lipschitz constant of $\phi$, we obtain, for such $\theta$,

$$
\begin{aligned}
\left\|\bar{z}_{n j}\left(t_{n, j-1}+\theta\right)-\bar{z}_{n, j-1}\left(t_{n, j-2}+\theta\right)\right\| & =\left\|\phi\left(t_{n, j-1}+\theta\right)-\phi\left(t_{n, j-2}+\theta\right)\right\| \\
& \leqslant L_{0}\left|t_{n, j-1}-t_{n, j-2}\right|=L_{0} h .
\end{aligned}
$$

(b) For $\theta \in\left(-t_{n, j-2}, 0\right]$, we have

$$
t_{n, j-1}+\theta \in\left(t_{n, j-1}-t_{n, j-2}, t_{n, j-1}\right] \text { and } t_{n, j-2}+\theta \in\left(0, t_{n, j-2}\right] \text {. }
$$

For such $\theta$,

$$
\left\|\bar{z}_{n j}\left(t_{n, j-1}+\theta\right)-\bar{z}_{n, j-1}\left(t_{n, j-2}+\theta\right)\right\| \leqslant \max _{1 \leqslant k \leqslant j}\left\|z_{n k}-z_{n, k-1}\right\| .
$$

(c) For $\theta \in\left(-t_{n, j-1},-t_{n, j-2}\right]$,

$$
t_{n, j-1}+\theta \in(0, h] \text { and } t_{n, j-2}+\theta \in(-h, 0] \text {. }
$$

For $\operatorname{such} \theta$,

$$
\begin{aligned}
\| \bar{z}_{n j}\left(t_{n, j-1}+\theta\right) & -\bar{z}_{n, j-1}\left(t_{n, j-2}+\theta\right) \| \\
\leqslant & \left\|\bar{z}_{n j}\left(t_{n, j-1}+\theta\right)-\bar{z}_{n j}(0)\right\|+\left\|\bar{z}_{n j}(0)-\bar{z}_{n, j-1}(0)\right\| \\
& \quad+\left\|\bar{z}_{n, j-1}(0)-\bar{z}_{n, j-1}\left(t_{n, j-2}+\theta\right)\right\| \\
\leqslant & \left\|z_{n 1}-z_{n 0}\right\|+\left\|\phi(0)-\phi\left(t_{n, j-2}+\theta\right)\right\| \\
\leqslant & \left\|z_{n 1}-z_{n 0}\right\|+L_{0} h .
\end{aligned}
$$

Case 3. $t_{n, j-1} \geqslant r, t_{n, j-2} \leqslant r$.

(a) For $\theta \in\left[-r,-t_{n, j-2}\right]$,

$$
t_{n, j-1}+\theta \in[0, h] \text { and } t_{n, j-2}+\theta \in[-h, 0] \text {. }
$$

For such $\theta$, we have (as in Case 2(c) above)

$$
\left\|\bar{z}_{n j}\left(t_{n, j-1}+\theta\right)-\bar{z}_{n, j-1}\left(t_{n, j-2}+\theta\right)\right\| \leqslant\left\|z_{n 1}-z_{n 0}\right\|+L_{0} h .
$$

(b) For $\theta \in\left(-t_{n, j-2}, 0\right]$,

$$
t_{n, j-1}+\theta \in\left(t_{n, j-1}-t_{n, j-2}, t_{n, j-1}\right] \text { and } t_{n, j-2}+\theta \in\left(0, t_{n, j-2}\right] \text {. }
$$


For $\operatorname{such} \theta$,

$$
\left\|\bar{z}_{n j}\left(t_{n, j-1}+\theta\right)-\bar{z}_{n, j-1}\left(t_{n, j-2}+\theta\right)\right\|=\max _{1 \leqslant k \leqslant j}\left\|z_{n k}-z_{n, k-1}\right\| .
$$

From the above three cases we see that for all $j=2,3, \ldots, n$ and all $\theta \in[-r, 0]$ we have

$$
\left\|\bar{z}_{n j}\left(t_{n, j-1}+\theta\right)-\bar{z}_{n j}\left(t_{n, j-2}+\theta\right)\right\| \leqslant \max _{1 \leqslant k \leqslant j}\left\|z_{n k}-z_{n, k-1}\right\|+L_{0} h .
$$

Therefore,

$$
\sup _{\theta \in[-r .0]}\left\|\bar{z}_{n j}\left(t_{n, j-1}+\theta\right)-\bar{z}_{n j}\left(t_{n, j-2}+\theta\right)\right\| \leqslant \max _{1 \leqslant k \leqslant j}\left\|z_{n k}-z_{n, k-1}\right\|+L_{0} h .
$$

From (2.8) we now obtain

$$
\begin{aligned}
\max _{1 \leqslant k \leqslant j}\left\|z_{n k}-z_{n, k-1}\right\| / h \leqslant & \beta \max _{1 \leqslant k \leqslant j}\left\|z_{n k}-z_{n, k-1}\right\|+\beta L_{0} h \\
& +L_{2}(M) h+L_{1}(M) \max _{1 \leqslant k \leqslant j}\left\|z_{n k}-z_{n, k-1}\right\| \\
& +h L_{1}(M) K_{4}+\max _{1 \leqslant k \leqslant j-1}\left\|z_{n k}-z_{n, k-1}\right\| / h,
\end{aligned}
$$

which yields

$$
\begin{aligned}
\left(1 / h-\beta-L_{1}(M)\right) & \max _{1 \leqslant k \leqslant j}\left\|z_{n k}-z_{n, k-1}\right\| \\
& \leqslant\left(L_{2}(M)+\beta L_{0}+L_{1}(M) K_{4}\right) h+\max _{1 \leqslant k \leqslant j-1}\left\|z_{n k}-z_{n, k-1}\right\| / h .
\end{aligned}
$$

Let $p=1-\left(\beta+L_{1}(M)\right) h$. Then $p \in(0,1)$ by our assumption. If $K_{5}$ denotes the number $L_{2}(M)+\beta L_{0}+L_{1}(M) K_{4}$, then

$$
\begin{aligned}
\frac{p}{h} \max _{1 \leqslant k \leqslant j}\left\|z_{n k}-z_{n, k-1}\right\| \leqslant & h K_{5}+\frac{1}{h} \max _{1 \leqslant k \leqslant j-1}\left\|z_{n k}-z_{n, k-1}\right\| \\
\leqslant & h K_{5}+\frac{h K_{5}}{p}+\frac{1}{p h} \max _{1 \leqslant k \leqslant j-2}\left\|z_{n k}-z_{n, k-1}\right\| \\
& \vdots \\
\leqslant &
\end{aligned}
$$

Using (2.7) we obtain

$$
\frac{1}{h} \max _{1 \leqslant k \leqslant j}\left\|z_{n k}-z_{n, k-1}\right\| \leqslant h K_{5} \sum_{s=1}^{n} \frac{1}{p^{s}}+\frac{1}{p^{n} h}\left\|z_{n 1}-z_{n 0}\right\| \leqslant h K_{5} \sum_{s=1}^{n} \frac{1}{p^{s}}+\frac{1}{p^{n}} K_{3} .
$$

Since

$$
h \sum_{s=1}^{n} \frac{1}{p^{s}} \leqslant h \sum_{s=1}^{n} \frac{1}{p^{n}}=T /\left[1-\frac{\left(\beta+L_{1}(M)\right) T}{n}\right]^{n}
$$

and

$$
\lim _{n \rightarrow \infty}\left[1-\left(\beta+L_{1}(M)\right) T / n\right]^{-n}=e^{\left(\beta+L_{1}(M)\right) T}
$$


this implies there exist $n_{1} \geqslant n_{0}$ and $N>0$ such that, for $n \geqslant n_{1}$,

$$
\left\|z_{n j}-z_{n, j-1}\right\| / h \leqslant N \text { for every } j=1,2, \ldots, n \text {. }
$$

We now define the functions

$$
z^{n}(t)=\left\{\begin{array}{l}
\phi(t), \quad t \in[-r, 0], \\
z_{n, j-1}+\left(t-t_{n, j-1}\right)\left(z_{n j}-z_{n, j-1}\right) / h, \quad t \in\left[t_{n, j-1}, t_{n j}\right] .
\end{array}\right.
$$

It is easy to see that the sequence $\left\{z^{n}(t)\right\}, t \in[-r, T]$, is uniformly Lipschitz with Lipschitz constant $N_{1}=\max \left\{N, L_{0}\right\}$. Let

$$
x^{n}(t)=\bar{z}_{n n}(t), \quad t \in[-r, T],
$$

where $z_{n n}(t)$ is obtained from (2.3). Explicitly, we have

$$
z_{n n}(t)= \begin{cases}\phi(t), & t \in[-r, 0], \\ z_{n 1}, & t \in\left(0, t_{n 1}\right], \\ \vdots & \vdots \\ z_{n j}, & t \in\left(t_{n, j-1}, t_{n j}\right], \\ \vdots & \vdots \\ z_{n n}= & \left(A\left(t_{n, n-1}\right)+(1 / h) I\right)^{-1}\left(z_{n, n-1} / h+G\left(t_{n, n-1}, \bar{z}_{n n_{n, n-1}}\right)\right), \\ \quad & t \in\left(t_{n, n-1}, T\right] .\end{cases}
$$

The operators $A^{n}(t)$ and $G^{n}(t)$ are defined by

$$
\begin{aligned}
& A^{n}(0)=A(0) \phi(0), \\
& A^{n}(t)=A\left(t_{n, j-1}\right) z_{n j} \text { for } t_{n, j-1}<t \leqslant t_{n j}, \\
& G^{n}(t)=G\left(t_{n, j-1}, \bar{z}_{n j_{n, j-1}}\right) \text { for } t_{n, j-1}<t \leqslant t_{n j} .
\end{aligned}
$$

It is easy to check that the function $z^{n}(t)$ is strongly differentiable on $[0, T]$ except at a finite number of points at which the strong left derivative $\left(d^{-} / d t\right) z^{n}(t)$ exists. Thus, from (2.5) and (2.10) we obtain

$$
\left(d^{-} / d t\right) z^{n}(t)+A^{n}(t)=G^{n}(t), \quad t \in\left(t_{n, j-1}, t_{n j}\right] .
$$

We will show that $x^{n}(t)-z^{n}(t) \rightarrow 0$ as $n \rightarrow \infty$ uniformly on $[-r, T]$. In what follows, " $\rightarrow$ " (" - ") denotes strong (weak) convergence. For $t \in(0, T], t \in$ $\left(t_{n, j-1}, t_{n j}\right]$ for some $j=1,2, \ldots, n$. From the definition of $z^{n}(t)$ we have

$$
\begin{aligned}
\left\|x^{n}(t)-z^{n}(t)\right\| & =\left\|z_{n j}-z_{n, j-1}-\left(t-t_{n, j-1}\right)\left(z_{n j}-z_{n, j-1}\right) / h\right\| \\
& =\left\|\left[h-\left(t-t_{n, j-1}\right)\right]\left(z_{n j}-z_{n, j-1}\right) / h\right\| \\
& \leqslant 2 h\left\|\left(z_{n j}-z_{n, j-1}\right) / h\right\| \leqslant 2 N h .
\end{aligned}
$$

Since $x^{n}(t)=z^{n}(t)=\phi(t)$ on [-r,0], our assertion follows.

THEOREM 2.3. The sequence of functions $\left\{x^{n}(t)\right\}, t \in[0, T]$, converges uniformly, as $n \rightarrow \infty$, to a strongly continuous function $u(t)$. Moreover, $u(t) \in D$ for every $t \in[0, T]$, 
$A(t) u(t)$ is weakly continuous, and the strong derivative $u^{\prime}(t)$ exists and equals $-A(t) u(t)+G\left(t, u_{t}\right)$ a.e. on $[0, T]$.

Proof. We first show that $z^{n}(t)$ converges uniformly as $n \rightarrow \infty$ to a (strongly continuous) function $u(t)$. Then it follows from above that $x^{n}(t)$ also converges uniformly to $u(t)$. Let $\left\{t_{n j}\right\}$ and $\left\{t_{m k}\right\}$ be two partitions of $[0, T]$, where

$$
t_{n j}=j T / n, j=0,1, \ldots, n, \quad t_{m k}=k T / m, k=0,1, \ldots, m .
$$

Let $t \in\left(t_{m, k-1}, t_{m k}\right] \cap\left(t_{n, j-1}, t_{n j}\right]$. By the Lipschitz continuity of $z^{n}(t)$ and Lemma 1.3 of Kato [12], we have

$$
\begin{aligned}
\left(d^{-} / d t\right) \| z^{m}(t) & -z^{n}(t) \|^{2} \\
= & 2\left\langle\left(d^{-} / d t\right) z^{m}(t)-\left(d^{-} / d t\right) z^{n}(t), J\left(z^{m}(t)-z^{n}(t)\right)\right\rangle \\
\leqslant & 2\left\langle G^{m}(t)-G^{n}(t)-A^{m}(t)+A^{n}(t), J\left(z^{m}(t)-z^{n}(t)\right)\right\rangle \\
\leqslant & 2\left\|G^{m}(t)-G^{n}(t)\right\|\left\|z^{m}(t)-z^{n}(t)\right\| \\
& -2\left\langle A^{m}(t)-A^{n}(t), J\left(z^{m}(t)-z^{n}(t)\right)\right\rangle .
\end{aligned}
$$

We also have

$$
\begin{aligned}
& \left\|G^{m}(t)-G^{n}(t)\right\|=\left\|G\left(t_{m, k-1}, \bar{z}_{m k_{t_{m, k-1}}}\right)-G\left(t_{n, j-1}, \bar{z}_{n j_{j_{n, j}, 1}}\right)\right\| \\
& \leqslant\left\|G\left(t_{m, k-1}, \bar{z}_{m k_{t_{m, k-1}}}\right)-G\left(t_{n, j-1}, \bar{z}_{m k_{t_{m, k}},}\right)\right\| \\
& +\left\|G\left(t_{n, j-1}, \bar{z}_{m k_{t_{m, k-1}}}\right)-G\left(t_{n, j-1}, \bar{z}_{m k_{t_{n, j}-1}}\right)\right\| \\
& +\left\|G\left(t_{n, j-1}, \bar{z}_{m k_{t_{n, j-1}}}\right)-G\left(t_{n, j-1}, \bar{z}_{n j_{t_{n,-1}}}\right)\right\| \text {, } \\
& \left\|G\left(t_{m, k-1}, \bar{z}_{m k_{t_{m, k-1}}}\right)-G\left(t_{n, j-1}, \bar{z}_{m k_{t^{\prime}, k-1}}\right)\right\| \leqslant L_{2}\left(\left\|\bar{z}_{m k_{t_{m}, k}}\right\|\right)\left|t_{m, k-1}-t_{n, j-1}\right|, \\
& \left\|G\left(t_{n, j-1}, \bar{z}_{m k_{i_{n, j-1}}}\right)-G\left(t_{n, j-1}, \bar{z}_{n j_{i_{n, j-1}}}\right)\right\| \leqslant \beta\left\|\bar{z}_{m k_{i_{n, j}-1}}-\bar{z}_{n j_{i_{n, j-1}}}\right\| \mathrm{PC} \\
& =\beta \sup _{s \in\left[t_{n, j-1}-r, t_{n, j-1}\right]}\left\|\bar{z}_{m k}(s)-\bar{z}_{n j}(s)\right\| \leqslant \beta \sup _{s \in[-r .1]}\left\|\bar{z}_{m k}(s)-\bar{z}_{n j}(s)\right\|, \\
& \left\|G\left(t_{n, j-1}, \bar{z}_{m k_{t_{m, k-1}}}\right)-G\left(t_{n, j-1}, \bar{z}_{m k_{t_{n, j-1}}}\right)\right\| \leqslant \beta\left\|\bar{z}_{m k_{i_{m, k}},}-\bar{z}_{m k_{t_{n, j-1}}}\right\| \mathrm{PC} .
\end{aligned}
$$

If $t_{n, j-1}<t_{m, k-1}$, then

$$
\bar{z}_{m k}\left(t_{m, k-1}+\theta\right)=\bar{z}_{m m}\left(t_{m, k-1}+\theta\right) \text { and } \bar{z}_{m k}\left(t_{n, j-1}+\theta\right)=\bar{z}_{m m}\left(t_{n, j-1}+\theta\right)
$$

for any $\theta \in[-r, 0]$. Also, since $x^{m}(t)-z^{m}(t) \rightarrow 0$ uniformly on $[-r, T]$, there exists a sequence of positive numbers $\varepsilon_{m}$ such that $\varepsilon_{m} \rightarrow 0$ as $m \rightarrow \infty$ and

$$
\begin{aligned}
\left\|\bar{z}_{m k}\left(t_{m, k-1}+\theta\right)-\bar{z}_{m k}\left(t_{n, j-1}+\theta\right)\right\| & =\left\|\bar{z}_{m m}\left(t_{m, k-1}+\theta\right)-\bar{z}_{m m}\left(t_{n, j-1}+\theta\right)\right\| \\
& =\left\|x^{m}\left(t_{m, k-1}+\theta\right)-x^{m}\left(t_{n, j-1}+\theta\right)\right\| \\
& \leqslant\left\|z^{m}\left(t_{m, k-1}+\theta\right)-z^{m}\left(t_{n, j-1}+\theta\right)\right\|+\varepsilon_{m} \\
& \leqslant N_{1}\left|t_{m, k-1}-t_{n, j-1}\right|+\varepsilon_{m}
\end{aligned}
$$


by the Lipschitz continuity of $z^{m}(t)$ on $[-r, T]$. Now, it is easy to prove that the sequence $t_{m, k-1}-t_{n, j-1}$ converges to zero uniformly in $j, k$. From this fact it follows that

$$
\left\|\bar{z}_{m k_{t_{m, k-1}}}-\bar{z}_{m k_{t_{n, j-1}}}\right\|_{\mathrm{PC}} \leqslant \bar{\varepsilon}_{m n}
$$

where $\bar{\varepsilon}_{m n} \rightarrow 0$ as $m, n \rightarrow \infty$. Since a similar inequality holds if $t_{m, k-1}<t_{n, j-1}$, we conclude that there exist sequences $\varepsilon_{m n}^{\prime}, \varepsilon_{m n}^{\prime \prime}$ such that $\varepsilon_{m n}^{\prime} \rightarrow 0$ and $\varepsilon_{m n}^{\prime \prime} \rightarrow 0$ as $m, n \rightarrow \infty$, and

$$
\begin{aligned}
\left\|G^{m}(t)-G^{n}(t)\right\| \leqslant & L_{2}(M)\left|t_{m . k-1}-t_{n, j-1}\right| \\
& +\beta \sup _{s \in[-r, t]}\left\|\bar{z}_{m k}(s)-\bar{z}_{n j}(s)\right\|+\beta \varepsilon_{m n}^{\prime} \\
\leqslant & \left(L_{2}(M)+\beta\right) \varepsilon_{m n}^{\prime \prime}+\beta \sup _{s \in[-r, t]}\left\|\bar{z}_{m k}(s)-\bar{z}_{n j}(s)\right\| .
\end{aligned}
$$

Now, for any $s \in[-r, t]$, we have

$$
\begin{aligned}
\left\|\bar{z}_{m k}(s)-\bar{z}_{n j}(s)\right\| & =\left\|\bar{z}_{m m}(s)-\bar{z}_{n n}(s)\right\|=\left\|x^{m}(s)-x^{n}(s)\right\| \\
& \leqslant\left\|z^{m}(s)-z^{n}(s)\right\|+\left\|\varepsilon_{m n}^{*}(s)\right\|,
\end{aligned}
$$

where $\varepsilon_{m n}^{*}(s) \rightarrow 0$ uniformly on $[-r, T]$ as $m, n \rightarrow \infty$. Thus,

$$
\sup _{s \in[-r, t]}\left\|\bar{z}_{m k}(s)-\bar{z}_{n j}(s)\right\| \leqslant \sup _{s \in[-r, l]}\left\|z^{m}(s)-z^{n}(s)\right\|+\varepsilon_{m n}^{*},
$$

where the constants $\varepsilon_{m n}^{*} \rightarrow 0$ as $m, n \rightarrow \infty$. Applying the above bounds to (2.13), we arrive at

$$
\begin{gathered}
\left(d^{-} / d t\right)\left\|z^{m}(t)-z^{n}(t)\right\|^{2} \\
\leqslant 2\left[\varepsilon_{m n}^{*}+\beta \sup _{s \in[-r, t]}\left\|z^{m}(s)-z^{n}(s)\right\|\right]\left\|z^{m}(t)-z^{n}(t)\right\| \\
-2\left\langle A^{m}(t)-A^{n}(t), J\left(z^{m}(t)-z^{n}(t)\right)\right\rangle
\end{gathered}
$$

where $\varepsilon_{m n}^{* *} \rightarrow 0$ as $m, n \rightarrow \infty$. Using the uniform continuity of $J$ on bounded subsets of $X$, we obtain a sequence of functions $\varepsilon_{m n}^{1}(t)$ with values in $X^{*}$ such that $\lim _{m, n \rightarrow \infty} \varepsilon_{m n}^{1}(t)=0$ uniformly on $[0, T]$ and

$$
J\left(z^{m}(t)-z^{n}(t)\right)=J\left(x^{m}(t)-x^{n}(t)\right)+\varepsilon_{m n}^{1}(t) .
$$

Thus,

$$
\begin{aligned}
&-\left\langle A^{m}(t)-A^{n}(t), J\left(z^{m}(t)-z^{n}(t)\right)\right\rangle \\
&=-\left\langle A\left(t_{m, k-1}\right) z_{m k}-A\left(t_{n, j-1}\right) z_{n j}, J\left(z^{m}(t)-z^{n}(t)\right)\right\rangle \\
&=-\left\langle A\left(t_{n, j-1}\right) z_{m k}-A\left(t_{n, j-1}\right) z_{n j}, J\left(x^{m}(t)-x^{n}(t)\right)\right\rangle \\
&-\left\langle A\left(t_{n, j-1}\right) z_{m k}-A\left(t_{n, j-1}\right) z_{n j}, \varepsilon_{m n}^{1}(t)\right\rangle \\
&-\left\langle A\left(t_{m, k-1}\right) z_{m k}-A\left(t_{n, j-1}\right) z_{m k}, J\left(z^{m}(t)-z^{n}(t)\right)\right\rangle \\
&=-\left\langle A\left(t_{n, j-1}\right) x^{m}(t)-A\left(t_{n, j-1}\right) x^{n}(t), J\left(x^{m}(t)-x^{n}(t)\right)\right\rangle \\
&-\left\langle A\left(t_{n, j-1}\right) z_{m k}-A\left(t_{n, j-1}\right) z_{n j}, \varepsilon_{m n}^{1}(t)\right\rangle \\
&-\left\langle A\left(t_{m, k-1}\right) z_{m k}-A\left(t_{n, j-1}\right) z_{m k}, J\left(z^{m}(t)-z^{n}(t)\right)\right\rangle,
\end{aligned}
$$


which, by the accretiveness of $A\left(t_{n, j-1}\right)$, implies

$$
\begin{aligned}
-\left\langle A^{m}(t)\right. & \left.-A^{n}(t), J\left(z^{m}(t)-z^{n}(t)\right)\right\rangle \\
\leqslant & {\left[\left\|A\left(t_{n, j-1}\right) z_{m k}\right\|+\left\|A\left(t_{n, j-1}\right) z_{n j}\right\|\right]\left\|\varepsilon_{m n}^{1}(t)\right\| } \\
& +\left|t_{m, k-1}-t_{n, j-1}\right| L_{1}\left(\left\|z_{m k}\right\|\right)\left(1+\left\|A\left(t_{m, k-1}\right) z_{m k}\right\|\right)\left\|z^{m}(t)-z^{n}(t)\right\| .
\end{aligned}
$$

From equation (2.5), we obtain

$$
\left\|A\left(t_{n, j-1}\right) z_{n j}\right\| \leqslant N+\beta M+K_{2} \leqslant K_{6} .
$$

Similarly, $\left\|A\left(t_{m, k-1}\right) z_{m k}\right\| \leqslant K_{6}$. Furthermore,

$$
\begin{aligned}
\left\|A\left(t_{n, j-1}\right) z_{m k}\right\| \leqslant & \left\|A\left(t_{n, j-1}\right) z_{m k}-A\left(t_{m, k-1}\right) z_{m k}\right\|+\left\|A\left(t_{m, k-1}\right) z_{m k}\right\| \\
\leqslant & \left|t_{n, j-1}-t_{m, k-1}\right| L_{1}\left(\left\|z_{m k}\right\|\right)\left(1+\left\|A\left(t_{m, k-1}\right) z_{m k}\right\|\right) \\
& +\left\|A\left(t_{m, k-1}\right) z_{m k}\right\| \\
\leqslant & 2 T L_{1}(M)\left(1+K_{6}\right)+K_{6}=K_{7} .
\end{aligned}
$$

Applying the above inequalities to (2.14), we obtain

$$
\left(d^{-} / d t\right)\left\|z^{m}(t)-z^{n}(t)\right\|^{2} \leqslant \varepsilon_{m n}+2 \beta \sup _{s \in[-r . t]}\left\|z^{m}(s)-z^{n}(s)\right\|^{2},
$$

where the sequence of positive constants $\varepsilon_{m n} \rightarrow 0$ as $m, n \rightarrow \infty$. Integrating (2.15), we obtain

$$
\left\|z^{m}(t)-z^{n}(t)\right\|^{2} \leqslant \varepsilon_{m n} T+2 \beta \int_{0}^{t}\left[\sup _{\tau \in[-r . s]}\left\|z^{m}(\tau)-z^{n}(\tau)\right\|\right]^{2} d s,
$$

where we have used $z^{m}(0)=z^{n}(0)=\phi(0)$. Since for any $t_{1}$ in the interval $[0, t]$, $t_{1} \in\left(t_{n, j-1}, t_{n j}\right] \cap\left(t_{m, k-1}, t_{m k}\right]$, for some $m, n, j, k$, we have

$$
\begin{aligned}
\left\|z^{m}\left(t_{1}\right)-z^{n}\left(t_{1}\right)\right\|^{2} & \leqslant \varepsilon_{m n} T+2 \beta \int_{0}^{t_{1}} \sup _{\tau \in[-r, s]}\left\|z^{m}(\tau)-z^{n}(\tau)\right\|^{2} d s \\
& \leqslant \varepsilon_{m n} T+2 \beta \int_{0}^{t} \sup _{\tau \in[-r . s]}\left\|z^{m}(\tau)-z^{n}(\tau)\right\|^{2} d s .
\end{aligned}
$$

We actually get

$$
\sup _{\tau \in[-r, t]}\left\|z^{m}(\tau)-z^{n}(\tau)\right\|^{2} \leqslant \varepsilon_{m n} T+2 \beta \int_{0}^{t}\left[\sup _{\tau \in[-r, s]}\left\|z^{m}(\tau)-z^{n}(\tau)\right\|\right]^{2} d s .
$$

An application of Gronwall's inequality above shows that the sequence $z^{m}(t)-$ $z^{n}(t) \rightarrow 0$ as $m, n \rightarrow \infty$ uniformly on $[-r, T]$. This implies that $z^{n}(t) \rightarrow u(t)$ uniformly on $[-r, T]$, where $u(t)$ is a strongly continuous function. Furthermore, since each $z^{n}(t)$ is Lipschitz continuous on $[-r, T]$ with Lipschitz constant $N$ on the interval $[0, T]$, the same fact is true for $u(t)$. In order to show that $u(t) \in D$ for all 
$t \in[0, T]$ and $A^{n}(t)-A(t) u(t)$, we first observe that $A^{n}(t)=A\left(t_{n, j-1}\right) z_{n j}=$ $A\left(t_{n, j-1}\right) x^{n}(t)$ for every $t$ in the interval $\left(t_{n, j-1}, t_{n j}\right]$. For such $t$ 's we have

$$
\begin{aligned}
\| A\left(t_{n, j-1}\right) & x^{n}(t)-A(t) x^{n}(t) \| \\
& \leqslant\left|t_{n, j-1}-t\right| L_{1}\left(\left\|x^{n}(t)\right\|\right)\left(1+\left\|A\left(t_{n, j-1}\right) x^{n}(t)\right\|\right) \\
& \leqslant L_{1}\left(\left\|x^{n}(t)\right\|\right)\left(1+K_{6}\right)\left|t_{n, j-1}-t\right| \leqslant N_{2}\left|t_{n, j-1}-t\right| \leqslant N_{2} T / n,
\end{aligned}
$$

which implies that $A^{n}(t)-A(t) x^{n}(t) \rightarrow 0$ uniformly on $[0, T]$ and $A(t) x^{n}(t)$ is uniformly bounded there. Since $x^{n}(t) \rightarrow u(t)$ and $A(t) x^{n}(t)$ is uniformly bounded, Lemma 2.5 of Kato [12] implies that $u(t) \in D$ and $A(t) x^{n}(t) \rightarrow A(t) u(t)$ for every $t \in[0, T]$. Now, since

$$
A^{n}(t)-A(t) u(t)=A\left(t_{n, j-1}\right) x^{n}(t)-A(t) x^{n}(t)+A(t) x^{n}(t)-A(t) u(t),
$$

we have actually shown that $A^{n}(t) \rightarrow A(t) u(t)$ for each $t$. The weak continuity of $A(t) u(t)$ can now be established as in Lemma 4.4 of Kato [12]. In order to show that $u(t)$ is weakly continuously differentiable on $[0, T]$, we observe first that, for every $f \in X^{*}$,

$$
\left\langle z^{n}(t), f\right\rangle=\langle\phi(0), f\rangle-\int_{0}^{t}\left\langle A^{n}(s), f\right\rangle d s+\int_{0}^{t}\left\langle G^{n}(s), f\right\rangle d s .
$$

We also observe that, for any $\theta \in[-r, 0]$,

$$
\bar{z}_{n j}\left(t_{n, j-1}+\theta\right)=x^{n}\left(t_{n, j-1}+\theta\right) \rightarrow u(s+\theta) \text { for } t_{n, j-1}<s \leqslant t_{n j} .
$$

Since

$$
G^{n}(s)=G\left(t_{n, j-1}, \bar{z}_{n j_{t_{n, j-1}}}\right)
$$

for such $s$, we find that $G^{n}(s) \rightarrow G\left(s, u_{s}\right)$ as $n \rightarrow \infty$. Since $\left\|A^{n}(t)\right\| \leqslant K_{6}$ and $\left\|G^{n}(t)\right\| \leqslant \beta M+K_{2}$, applying Lebesgue's bounded convergence theorem, we obtain

$$
\langle u(t), f\rangle=\langle\phi(0), f\rangle-\int_{0}^{t}\left[\langle A(s) u(s), f\rangle-\left\langle G\left(s, u_{s}\right), f\right\rangle\right] d s .
$$

Since the integrand above is continuous in $t$, we have shown that $\langle u(t), f\rangle$ is continuously differentiable on $[0, T]$. The proof that the strong derivative of $u(t)$ exists a.e. and equals $-A(t) u(t)+G\left(t, u_{t}\right)$ follows as in Kato [12, Lemma 4.6] and is therefore omitted.

3. Concluding remarks - an example. All of our results are also valid for the infinite delay version of (FDE). That is, we can let PC be the space of locally piecewise continuous functions $f:(-\infty, 0] \rightarrow X$ which are bounded. If, in addition, we replace $[-r, 0]$ and $[-r, T]$ by $(-\infty, 0]$ and $(-\infty, T]$, respectively, then a straightforward modification of the proof of the above theorem yields the analogous result.

We mentioned in the introduction that since the perturbed evolution equation (PE) is a special case of (FDE), we can use our method to approximate the solutions of (PE). This is a substantial improvement of the analogous result of Kartsatos and Zigler [11] in the following sense: our scheme converges without assuming that $A(0)$, 
hence $A(t)$, maps bounded subsets of $D$ into bounded subsets of $X$. However, it is important to mention here the fact that, in the case of (PE), we should consider instead the method developed in [11] which starts with the equation

$$
z_{n j}=\left(A\left(t_{n j}\right)+(1 / h) I\right)^{-1}\left(z_{n, j-1} / h+G\left(t_{n j}, z_{n j}\right)\right) \text {. }
$$

In this case we need not introduce the functions $\bar{z}_{n j}$, and the method converges again without the boundedness of $A(0)$ and with $G:[0, T] \times X \rightarrow X$ a global Lipschitzian-like function.

In a forthcoming paper the authors are planning to study the existence of solutions of (FDE) for perturbations $G$ which are "local Lipschitzians" in their second variable. It is hoped that an analogous method of lines can be developed for such problems.

Even in the case $X=R^{n}$, our result appears to offer a new method for the solutions of (FDE). Unfortunately, the results of Cryer and Tavernini [4, Theorem 4.1] do not apply to (FDE) because the mapping $F(t, u, v)=-A(t) u+G(t, v)$ is not defined on $X \times X$ with respect to $(u, v)$. Thus, it is impossible to construct approximating sequences of functions as in [4].

As an example we cite the functional "heat" equation discussed in [10]:

$$
u_{t}-a(t) k\left(u_{x}\right) u_{x x}=f(t, u(x, t-r))
$$

for $r>0, t \in[0, T], x \in(0,1)$, with boundary conditions

$$
\begin{array}{ll}
u(x, \theta)=\phi(x, \theta), & -r \leqslant \theta \leqslant 0 \\
u(0, t)=\alpha u^{\prime}(0, t), & 0 \leqslant t \leqslant T, \\
u(1, t)=-\beta u^{\prime}(1, t), & 0 \leqslant t \leqslant T .
\end{array}
$$

Here we let $X=L^{p}[0,1]$, for some $p \in(1, \infty)$, and $\tilde{A}(t): D \subset X \rightarrow X$ be such that

$$
(\tilde{A}(t) u)(x)=-a(t) k\left(u_{x}(t, x)\right) u_{x x}(t, x)
$$

and

$$
D=\left\{u \in L^{p}[0,1] ; u \in C^{2}[0,1], u(0)=\alpha u^{\prime}(0), \quad u(1)=-\beta u^{\prime}(1)\right\} .
$$

For $\psi \in \mathrm{PC}$, we define $G(t, \psi)=f(t, \psi(-r))$. Further conditions on $a, k, f$ and the constants $\alpha, \beta$ can be given (cf. [10]) so that conditions (A.1)-(A.5) are satisfied for the closure $A(t)$ of $\tilde{A}(t)$ in $L^{p}[0,1]$. Thus, the method of lines developed above can be applied to the abstract version (FDE) of (3.1).

\section{REFERENCES}

1. H. Banks and J. Burns, Hereditary control problems: mumerical methods hased on aleraging approximations. SIAM J. Control Optim. 16 (1978), 169-208.

2. H. Banks and F. Kappel, Spline approximations for functional differential equations. J. Differential Equations 34 (1974), 496-522.

3. M. Crandall and A. Pazy, Nonlinear evolution equations in Banach spaces. Israel J. Math. 11 (1972). $57-94$.

4. C. Cryer and L. Tavernini. The numerical solution of Volterra differential equations hy Euler's method, SIAM J. Numer. Anal. 9 (1972), 105-129.

5. J. Dyson and R. Villella Bressan, Functional differential equations and nonlinear evolution operators, Proc. Roy. Soc. Edinburgh Sect. A 75 (1975/76). 223-234.

6. Semigroups of translations associated with functional and functional differential equations, Proc. Roy. Soc. Edinburgh Sect. A 82 (1979), 171-188. 
7. L. Evans, Nonlinear evolution equations in an arbitran Banach space, Israel J. Math. 26 (1977). 1-42.

8. W. Fitzgibbon, Approximations of nonlinear evolution equations, J. Math. Soc. Japan 25 (1973). 211-221.

9. F. Kappel and W. Schappacher, Non-linear functional differential equations and abstract integral equations, Proc. Roy. Soc. Edinburgh Sect. A 84 (1979), 71-91.

10. A. G. Kartsatos and M. E. Parrott. Convergence of the Kato approximants for evolution equations involving functional perturbations. J. Differential Equations 47 (1983). 358-377.

11. A. G. Kartsatos and W. Zigler, Rothe's method and weak solutions of perturbed evolution equations in reflexive Bunach spaces, Math. Ann. 219 (1976), 159-166.

12. T. Kato, Nonlinear semigroups and evolution equations, J. Math. Soc. Japan 19 (1967), 508-520.

13. J. Mermin, Accretive operators and nonlinear semigroups, Ph.D. Thesis, Univ. of California. Berkeley, 1968.

14. J. Nečas, Application of Rothe's method to abstract parabolic equations, Czechoslovak Math. J. 24 (1974), 496-500.

15. M. E. Parrott. Representation and approximation of generalized solutions of a nonlinear functional differential equation, Nonlinear Anal. 6 (1982), 307-318.

16. E. Rothe. Zweidimensionale parabolische Randwertaufgaben als Grenzfall eindimensionaler Randwertaufgaben, Math. Ann. 102 (1930), 650-670.

17. R. Thompson, On some functional differential equations: Existence of solutions and difference approximations, SIAM J. Numer. Anal. 5 (1968), 475-487.

18. G. Webb, Asymptotic stability for abstract nonlinear functional differential equations, Proc. Amer. Math. Soc. 54 (1976), 225-230.

19. Autonomous nonlinear functional differential equations and nonlinear semigroups. J. Math. Anal. Appl. 46 (1974), 1-12.

Department of Mathematics, University of South Florida, Tampa, Florida 33620 